\title{
Anabases
}

ANABASES Traditions et réceptions de l'Antiquité

$14 \mid 2011$

Varia

\section{Mommsen, Cesare e il cesarismo}

\section{Leandro Polverini}

\section{OpenEdition}

\section{Journals}

Edizione digitale

URL: http://journals.openedition.org/anabases/2838

DOI: 10.4000/anabases.2838

ISSN: 2256-9421

\section{Editore}

E.R.A.S.M.E.

\section{Edizione cartacea}

Data di pubblicazione: 1 ottobre 2011

Paginazione: 173-184

ISSN: 1774-4296

\section{Notizia bibliografica digitale}

Leandro Polverini, « Mommsen, Cesare e il cesarismo », Anabases [Online], 14 | 2011, Messo online il

01 octobre 2014, consultato il 20 octobre 2019. URL : http://journals.openedition.org/anabases/2838 ; DOI : 10.4000/anabases.2838 
Anabases 14 (2011), p. 173-184.

\section{Mommsen, Cesare e il cesarismo*}

LeANDro Polverini

Il più grande storico di Roma del XIX secolo fu - oltre che uno studioso (ed un organizzatore di imprese scientifiche) di eccezionale importanza - personaggio significativo nella vita politica tedesca, prima e dopo la fondazione del secondo Reich, e scrittore di forte rilievo ${ }^{1}$. Di Mommsen come studioso, basterà ricordare il dato statistico più ovvio (e più imponente): gli oltre 1500 titoli dell'elenco dei suoi scritti ${ }^{2}$. Di Mommsen

* Il testo presentato a Tolosa il 18 marzo 2011, in francese, si pubblica in italiano con l'aggiunta di un apparato di note e l'omissione di quanto aveva interesse occasionale. $\mathrm{Ma}$ non era occasionale il sentimento che ispirava - e ispira - l'espressione dei ringraziamenti: a Corinne Bonnet e a Pascal Payen per la dedica del numero 12 di Anabases e l'invito alla sua presentazione; agli autori dei contributi dell'omaggio nella rivista; ai colleghi e agli studenti che parteciparono alla presentazione, in particolare a quelli venuti dall'Italia, dal direttore dell'École française de Rome, Michel Gras, a Gino Bandelli dell'Università di Trieste, ad alcuni allievi di Roma Tre.

1 Una sintesi dei tre fondamentali aspetti di Theodor Mommsen (Garding, Schleswig, 30.11.1817 - Charlottenburg, 1.11.1903) è già nel titolo di una recente raccolta di saggi: J. WiesehöFer (Hg.), Theodor Mommsen: Gelehrter, Politiker und Literat, Stuttgart, Steiner, 2005. La bibliografia su Mommsen è immensa; tanto più notevole si rivela il quadro d'assieme, dell'uomo e della sua opera, di S. ReBenich, Theodor Mommsen. Eine Biographie, München, Beck, 2002, $2007^{2}$.

2 K. Zangemeister, Theodor Mommsen als Schriftsteller. Ein Verzeichnis seiner Schriften, bearbeitet und fortgesetzt von E. Jacobs, neu bearbeitet von S. Rebenich, Hildesheim, Weidmann, 2000. L'edizione di Jacobs (Berlin, Weidmann, 1905) constava di 1513 numeri; altri 34 sono stati aggiunti da Rebenich nella nuova edizione (dove sono elencati a p. VIII). 
come uomo politico, è ben noto che egli stesso aveva rivelato la natura intimamente e profondamente politica della propria personalità nel famoso codicillo testamentario, la cui pubblicazione fu rinviata - et pour cause - dal 1933 al 1948: «Politische Stellung und politischen Einfluß habe ich nie gehabt und nie erstrebt; aber in meinem innersten Wesen, und ich meine, mit dem Besten was in mir ist, bin ich stets ein animal politicum gewesen und wünschte ein Bürger zu sein. Das ist nicht möglich in unserer Nation, bei der Einzelne, auch der Beste, über den Dienst im Gliede und den politischen Fetischismus nicht hinauskommt. Diese innere Entzweiung mit dem Volke, dem ich angehöre, hat mich durchaus bestimmt, mit meiner Persönlichkeit, soweit mir dies irgend möglich war, nicht vor das deutsche Publikum zu treten, vor dem mir die Achtung fehlt ${ }^{3}$ ». Di Mommsen come scrittore, infine, è significativo il riconoscimento più vistoso: l'assegnazione del premio Nobel per la letteratura, nel $1902^{4}$.

L'opera di Mommsen in cui lo studioso, l'uomo politico e lo scrittore convergono, e si fondono compiutamente, è la Römische Geschichte, cioè l'insieme organico e unitario dei primi tre volumi di una storia romana di carattere generale, apparsi fra il $1854 \mathrm{e}$ il $1856^{5}$ (trent'anni più tardi, il V volume avrebbe avuto altra genesi, altra ispirazione, altra natura ${ }^{6}$; il IV volume non fu mai pubblicato ${ }^{7}$ ). Alla Römische Geschichte faceva,

3 È il terzo dei cinque capoversi del codicillo testamentario datato «Heringsdorf, 2. September 1899", pubblicato nella rivista tedesca Die Wandlung 3 (1948), p. 69-70 (dalla quale è riprodotta la citazione), e subito anche nella rivista italiana Athenaeum 26 (1948), p. 285-287. Traduzione italiana, con importante commento: G. PASQUALI, «Il testamento di Teodoro Mommsen", Rivista storica italiana 61 (1949), p. 332-350 (poi in Stravaganze quarte e supreme, Venezia, Pozza, 1951, p. 147-163 = Pagine stravaganti, Firenze, Sansoni, 1968, II, p. 383-396); traduzione francese: A. AymARD, «Le codicille de Th. Mommsen", Revue des études anciennes 54 (1952), p. 213-214. Vd. ora ReBENICH, Theodor Mommsen, p. 165-193 e 256-260, con aggiornamento bibliografico a p. 234-235 della seconda edizione.

4 C. Lanza, «Il Nobel a Mommsen», Studia et documenta historiae et iuris 68 (2002), p. 501-525; H. SCHLANGE-SCHÖNINGEN, «Ein "goldener Lorbeerkranz" für die "Römische Geschichte". Theodor Mommsens Nobelpreis für Literatur", in WIESEHÖFER, Theodor Mommsen, p. 207-228.

5 Th. Mommsen, Römische Geschichte, I (Bis zur Schlacht von Pydna), II (Von der Schlacht von Pydna bis auf Sullas Tod), III (Von Sullas Tod bis zur Schlacht von Thapsus), Berlin, Weidmann, 1854, 1855, 1856. Lo straordinario successo dei tre volumi è dimostrato dalle otto edizioni che seguirono alla prima già durante la vita di Mommsen (nona edizione: 1902, 1903, 1904).

6 Come spiega l'autore nella premessa e nell'introduzione al volume: Th. MommsEn, Römische Geschichte, V (Die Provinzen von Caesar bis Diocletian), Berlin, Weidmann, 1885, $1904^{5}$ (la quinta edizione del $\mathrm{V}$ volume completava la nona edizione dei primi tre).

7 Vd. A. Demandt, «Einleitung», in Th. Mommsen, Römische Kaisergeschichte, nach den Vorlesungs-Mitschriften von S. und P. Hensel 1882/86, hrsg. von B. und A. Demandt, München, Beck, 1992, p. 15-56, in particolare 15-36. - Sull'opera di gran lunga più nota di Mommsen, anche perché la sola di carattere 'divulgativo', resta fondamentale il saggio di K. Christ, "Theodor Mommsen und die "Römische Geschichte"», in Th. Mommsen, 
in effetti, precipuo riferimento l'assegnazione del premio Nobel per la letteratura del $1902^{8}$. E di natura essenzialmente e appassionatamente politica era la caratteristica modernizzazione della storia romana repubblicana che affascinò subito i contemporanei, e continua ad affascinare anche oggi (come mostrano le continue ristampe del testo originale ${ }^{9}$, ma anche delle traduzioni ${ }^{10}$ ). Letteratura e politica non sovrastano, tuttavia, esaltano anzi la vigorosa originalità dell'impianto scientifico dell'opera, in ordine sia al quadro generale, sia a particolari in apparenza minuti, in realtà densi di significato storico ${ }^{11}$. Questo contributo intende, appunto, mostrare uno specifico esempio del nesso profondo e della reciproca interdipendenza, nei primi tre volumi della Römische Geschichte, fra i tre fondamentali aspetti della personalità di Mommsen.

Il III volume si concludeva cronologicamente con la battaglia di Tapso (6 aprile - in realtà, 7 febbraio ${ }^{12}$ - del 46 a. C.), cioè con la definitiva affermazione militare di Cesare, che avrebbe dovuto preludere alla sua definitiva affermazione politica e alla

Römische Geschichte, München, DTV, 1976 (20016), 8, p. 7-66 (poi in K. CHRIST, Römische Geschichte und Wissenschaftsgeschichte, III, Darmstadt, WBG, 1983, p. 26-73).

8 Vd. LanZA, «Il Nobel a Mommsen», p. 502-504; SCHLANGe-SCHÖNIngEN, «Ein "goldener Lorbeerkranz" für die "Römische Geschichte”", in particolare p. 207: «Der Sekretär teilte Mommsen weiterhin mit, daß ihm der Preis "unter besonderer Berücksichtigung" des "monumentalen Werkes Römische Geschichte" zugesprochen worden sei».

9 La fortunata edizione in otto volumi 'tascabili', a cura di K. Christ (vd. la precedente n. 7), è stata ora riproposta in due volumi, con introduzione di S. Rebenich (Darmstadt, WBG, 2010).

10 In Inghilterra, per esempio, la classica traduzione dei volumi I-III, di W. P. Dickson (London, Bentley, 1868), è stata parzialmente ripubblicata da C. J. Shepherd, con introduzione di A. Grafton (A History of Rome from the foundation of the City to the sole rule of Julius Caesar, London, The Folio Society, 2006). In Italia, la più recente di una numerosa serie di traduzioni, pubblicata con introduzione di G. Pugliese Carratelli (Storia di Roma antica, I-III, Firenze, Sansoni, 1960: il volume II corrisponde ai volumi II e III, il III al volume $\mathrm{V}$ dell'edizione originale), è stata parzialmente ristampata più volte, da ultimo nel 2001. In Francia, la traduzione dei volumi I-III e V, rispettivamente di Ch. A. Alexandre (in otto tomi: Paris, Franck, 1863-1872) e di R. Cagnat e J. Toutain (in tre tomi: Paris, Vieweg, poi Bouillon, 1887-1889), è stata ripubblicata in due volumi da C. Nicolet (Histoire romaine, Paris, Laffont, 1985 = Le Grand livre du mois, 1996).

11 Ricordo, per esempio, che sul problema allora molto dibattuto della presenza di Germani nella battaglia di Clastidium (222 a. C.) Mommsen non esitò a ribaltare la propria posizione sull'attendibilità della notizia dei Fasti triumphales dalla prima alla seconda edizione (uscita due anni dopo) del I volume: vd. L. PolverinI, «Germani in Italia prima dei Cimbri?», in B. e P. SCARDigli (cur.), Germani in Italia, Roma, CNR, 1994, p. 3 e n. 10-11.

12 Secondo il conguaglio di P. Groebe, «Der römische Kalender in den Jahren 65-43 v. Chr.», in W. Drumann, Geschichte Roms, III'2 Leipzig, Borntraeger, 1906 (= Hildesheim, Olms, 1964), p. 753-827, in particolare 818. 
conseguente sistemazione dell'impero mediterraneo. Nella prospettiva mommseniana, in quella almeno della Römische Geschichte, Cesare non è solo il più grande personaggio della storia romana; degli aspetti essenziali e più creativi della storia romana egli è, in certo modo, la personificazione assoluta (l'icona, diremmo oggi). Di qui l'appassionato e incondizionato entusiasmo con il quale parla della persona, delle idee, dell'opera di Cesare un Mommsen ancora romantico, a metà del XIX secolo: romantico in termini sia di sensibilità politica e letteraria, sia di concezione storiografica. In termini storiografici, appunto, è fondamentale (per quanto riguarda l'interpretazione mommseniana di Cesare) il capitolo XI del V libro ${ }^{13}$ : è, in sostanza, l'ultimo capitolo del III volume, perché il capitolo XII presenta una sintesi della vita religiosa e culturale, letteraria e artistica, del trentennio che va dalla morte di Silla alla battaglia di Tapso.

Die alte Republik und die neue Monarchie è il titolo che, con caratteristica efficacia, sottolinea il carattere conclusivo di un capitolo di straordinario, di sempre nuovo interesse ad ogni rilettura ${ }^{14}$. Si apre con un intenso profilo psicologico più che biografico di Cesare ("Caesars Charakter» $\left.{ }^{15}\right)$, già per natura - prima che per la sua formazione e le varie esperienze di vita - destinato ad essere uomo di Stato nel più profondo senso della parola («Caesar als Staatsmann»). E vero uomo di Stato Cesare si rivelò infatti, dopo Tapso, cercando innanzitutto di eliminare dalla scena politica i vecchi partiti, causa delle guerre civili («Beseitigung der alten Parteien»): così i populares, che ebbero non infondato motivo di ritenersi traditi dal loro capo ("Unzufriedenheit der Demokraten», «Caelius und Milo», "Dolabella»), come gli optimates («Maßregeln gegen Pompeianer und Republikaner»), solo in minima parte riconciliati dalla clemenza di Cesare e dalle sue misure di grazia («Amnestie»). La radicale, drastica posizione di Cesare nei confronti dei contrapposti partiti («Caesar gegenüber den Parteien»), in assoluto

13 La divisione dell'opera in volumi era dettata da ovvie esigenze di ordine editoriale; la divisione più propriamente storiografica è in libri, alla maniera della storiografia classica. Il V libro (che occupava l'intero III volume), Die Begründung der militärischen Monarchie, si propone come naturale, cioè inevitabile, conclusione storica dell'età dai Gracchi a Silla descritta nel IV libro (che occupava l'intero II volume), Die Revolution (il titolo avrebbe avuto singolare fortuna nel successivo secolo e mezzo di dibattito storiografico). - Il I volume conteneva tre libri (1. Bis zur Abschaffung des römischen Königthums, 2. Von der Abschaffung des römischen Königthums bis zur Einigung Italiens, 3. Von der Einigung Italiens bis auf die Unterwerfung Karthagos und der griechischen Staaten), il V volume un libro (8. Länder und Leute von Caesar bis Diocletian).

14 Vd. K. Christ, Caesar. Annäherungen an einen Diktator, München, Beck, 1994, p. 142-147 (nel quadro di un'analisi storico-storiografica del Cesare di Mommsen: p. 134-165).

15 La densa articolazione della trattazione mommseniana si lascia agilmente ripercorrere grazie ai titoletti marginali, opportunamente introdotti a partire dalla seconda edizione (dalla quale si citano). 
dispregio delle possibili conseguenze di ordine personale ${ }^{16}$, spiega appunto come egli intendesse rinnovare profondamente lo Stato ("Caesars Werk»).

Con la sezione dedicata all'opera di Cesare si entra nell'argomento specifico di questo contributo: è, dunque, opportuno proporla (almeno in parte) direttamente, seguendo lo svolgimento del pensiero di Mommsen attraverso le varie edizioni del III volume, in particolare dalla prima (1856) alla sesta $(1875){ }^{17}$. Questa era la formulazione originaria ${ }^{18}$ :

Versuchen wir im Einzelnen Rechenschaft zu geben von der Ueberführung der alten Zustände in die neue Bahn, so ist zunächst daran zu erinnern, daß Caesar nicht kam um anzufangen, sondern um zu vollenden. Der Plan zu einer neuen zeitgemäßen Politie ${ }^{19}$, längst von Gaius Gracchus entworfen, war von seinen Anhängern und Nachfolgern wohl mit mehr oder minder Geist und Glück, aber ohne Schwanken festhgehalten worden. Caesar, von Haus aus und gleichsam schon nach Erbrecht ${ }^{20}$ das Haupt der Popularpartei, hatte seit dreißig Jahren deren Schild hoch emporgehalten, ohne je die Farbe zu verleugnen ${ }^{21}$ oder auch nur zu decken; er blieb Demokrat auch als Monarch.

«Monarca democratico»: tale fu Cesare, secondo Mommsen ${ }^{22}$, non solo in quanto erede delle idealità e del programma (e dell'odio contro l'aristocrazia) del movimento 'democratico' iniziato da Gaio Gracco, ma per la natura stessa della sua monarchia («die Vertretung der Nation durch ihren höchsten und unumschränkten Vertrauensmann»), nella quale la 'democrazia' romana trovò compiuta realizzazione («zur Vollendung und Erfüllung gelangte»). Con le parole di Mommsen, nel séguito della citazione:

16 È caratteristica (per quanto riguarda, al tempo stesso, l'ispirazione politica e lo stile letterario di Mommsen) la conclusione della sezione: «Noch weniger fragte er, wer ihn haßte oder auf Mord gegen ihn sann. Wie jeder echte Staatsmann diente er dem Volke nicht um Lohn, auch nicht um den Lohn seiner Liebe, sondern gab die Gunst der Zeitgenossen hin für den Segen der Zukunft und vor allem für die Erlaubniß seine Nation retten und verjüngen zu dürfen» (così nella prima edizione del III volume, p. 442-443; il testo della citazione resta immutato nelle edizioni successive).

17 Ad esse si farà riferimento con il solo numero del volume e l'indicazione sia dell'edizione (in esponente), sia dell'anno di pubblicazione. - Nella presentazione del testo a Tolosa, avevo fatto opportuno ricorso alla bella traduzione di Charles Alexandre, citata nella precedente n. 10; va da sé che solo il riferimento al testo originale permette di seguire i continui, significativi interventi di Mommsen sulla sua opera (particolarmente ampi e sostanziali nella seconda edizione).

18 III $^{1}, 1856$, p. 443.

19 Poi (III 5 , 1869, p. 461): «Politik».

20 In quanto nipote di Mario (che aveva sposato una sorella del padre di Cesare).

21 Poi (III ${ }^{2}, 1857$, p. 457): «wechseln».

$22 \mathrm{Al}$ «monarca democratico» di Mommsen corrisponde almeno formalmente, nel linguaggio del XX secolo, il «dittatore democratico» di L. CANFora, Cesare. Il dittatore democratico, Roma-Bari, Laterza, 1999. 
Wie er die Erbschaft seiner Partei [...] unbeschränkt antrat, der Aristokratie und den echten Aristokraten den bittersten selbst persönlichen $\mathrm{Haß}$ zollte und die wesentlichen Gedanken der römischen Demokratie [... ${ }^{23}$ unverändert festhielt, so war auch seine Monarchie so wenig mit der Demokratie in Widerspruch, daß vielmehr diese erst durch $\operatorname{sie}^{24}$ zur Vollendung und Erfüllung gelangte. Denn diese Monarchie war nicht die orientalische Despotie von Gottes Gnaden, sondern die Monarchie, wie Gaius Gracchus sie gründen wollte, wie Perikles und Cromwell sie gründeten: die Vertretung der Nation durch ihren höchsten und unumschränkten Vertrauensmann ${ }^{25}$. Es waren insofern die Gedanken, die zum Werke Caesars zu Grunde lagen, nicht eigentlich neue; aber ihm gehört ihre Verwirklichung, die zuletzt überall die Hauptsache bleibt, und ihm die Großheit der Ausführung, die selbst den genialen Entwerfer, wenn er sie hätte schauen können, überrascht haben möchte ${ }^{26}$.

\section{3}

«Die Stellung des neuen Staatsoberhaupts erscheint formell in seltsamer Gestalt ${ }^{27}$ ", innanzitutto come dittatura ${ }^{28}$ : così, nella prima edizione del III volume, si passava

23 Cioè (dal tempo dei Gracchi): il miglioramento della condizione dei debitori, la colonizzazione transmarina, il progressivo livellamento delle differenze giuridiche all'interno delo Stato, l'emancipazione dal Senato del potere esecutivo.

24 Poi (III ${ }^{2}, 1857$, p. 457): «jene».

25 La formula compendia con vigorosa efficacia - in termini storici e politici - la natura della «monarchia democratica» di Mommsen (sulla quale CHRIsT, Caesar, p. 144-145).

26 Nella seconda edizione, la considerazione che conclude il passo citato era così completata: «und die Jeden, dem sie in lebendiger Wirklichkeit oder im Spiegel der Geschichte entgegengetreten ist, welcher geschichtlichen Epoche und welcher politischen Farbe immer er angehöre, je nach dem Maß seiner Fassungskraft für menschliche und geschichtliche Größe mit tiefer und tieferer Bewegung und Bewunderung ergriffen hat und ewig ergreifen wird" (III ${ }^{2}, 1857$, p. 457-458). - Anche un grande ammiratore di Mommsen come Charles Alexandre era indotto, da queste e simili espressioni entusiastiche (vd. la precedente n. 16), a rilevare «sa complaisante et excessive apothéose de César» e a prenderne le distanze: così a p. XI dell' "Avant-propos» dell'ultimo tomo della citata traduzione francese, steso peraltro nel clima politico e culturale seguito alla disfatta del 1870 .

27 III $^{1}, 1856$, p. 443.

28 Opportuna, dunque, la riformulazione introdotta da Mommsen in una successiva edizione: «Die Stellung des neuen Staatsoberhaupts erscheint formell, zunächst wenigstens, als Dictatur» (III ${ }^{6}, 1875$, p. 478; ivi, p. 479, la definizione costituzionale della dittatura di Cesare, che non è la dittatura arcaica, ovviamente, ma la dittatura rei publicae constituendae di Silla: «ein Amt, dessen Competenz nicht durch die verfassungsgemäßigen Ordnungen über das höchste Einzelamt, sondern durch besonderen Volksschluß festgestellt ward und zwar dahin, daß der Inhaber in dem Auftrag Gesetzte zu entwerfen und das Gemeinwesen zu ordnen eine rechtlich unumschränkte die republikanische Theilung der Gewalten aufhebende Amtsbefugniß empfing»). 
senz'altro alla successiva sezione del capitolo. Ma nella seconda edizione, fra la sezione "Caesars Werk» e la sezione «Formulirung der neuen Monarchie ${ }^{29}$ ", fu inserita un'ampia aggiunta ${ }^{30}$, di grande interesse generale e specifico. Anche in questo caso è, dunque, opportuno proporne direttamente la parte più significativa, articolando la compatta stesura secondo la sua forte scansione argomentativa:

Wohl aber wird es gerade hier am Orte sein das, was der Geschichtschreiber stillschweigend überall voraussetzt, einmal ausdrücklich zu fordern und Einspruch zu thun gegen die der Einfalt und der Perfidie gemeinschaftliche Sitte geschichtliches Lob und geschichtlichen Tadel von den gegebenen Verhältnissen abgelöst als allgemein gültige Phrase zu verbrauchen, in diesem Falle das Urtheil über Caesar in ein Urtheil über den sogenannten Caesarianismus ${ }^{31}$ umzudeuten.

Il "cosiddetto cesarismo» era, in effetti, un neologismo non attestato prima del 1850; resta classica la definizione che ne diede Littré: "Domination des Césars, c'est-àdire des princes ["dei dittatori”, diremmo oggi] portés au gouvernement par la démocratie mais revêtus d'un pouvoir absolu» (con una seconda accezione: «Théorie de ceux qui pensent [ce ne sono ancora!] que cette forme de gouvernement est la meilleure») ${ }^{32}$. Il vistoso intervento polemico di Mommsen, nell'aggiunta del 1857, richiamava con forza la "necessità di distinguere tra Cesare e Cesarismo» ${ }^{33}$, perché la storia del passato aiuta bensì a comprendere il presente, ma non nel senso in cui la sentenza historia magistra vitae viene comunemente intesa:

Freilich soll die Geschichte der vergangenen Jahrhunderte die Lehrmeisterin des laufenden sein; aber nicht in dem gemeinen Sinne, als könne man die Conjuncturen der Gegenwart in den Berichten über die Vergangenheit nur einfach wieder aufblättern und aus denselben der politischen Diagnose und Receptirkunst die Symptome und Specifica zusammenlesen; sondern sie ist lehrhaft einzig insofern, als die Beobachtung der älteren Culturen die organischen Bedingungen der Civilisation überhaupt, die überall gleichen Grundkräfte und die überall verschiedene Zusammensetzung dersel-

29 Poi, dalla sesta edizione (in conformità del nuovo incipit della sezione, citato nella nota precedente): «Dictatur».

30 III $^{2}, 1857$, p. 458-459. L'importanza che ad essa annetteva Mommsen è messa in evidenza già dalla sua estensione: quasi il doppio dell'estensione della sezione sull'opera di Cesare nella prima edizione.

31 Poi (III ${ }^{5}, 1869$, p. 462): «Caesarismus». Vd. la successiva n. 45.

32 É. LitTré, Dictionnaire de la langue française, I, Paris, Hachette, 1863, p. 534.

33 Così A. Momigliano, "Per un riesame della storia dell'idea di Cesarismo", Rivista storica italiana 68 (1956), p. 220-229, in particolare 223 (poi in Secondo contributo alla storia degli studi classici, Roma, Edizioni di Storia e Letteratura, 1960 [= 1984], p. 273-282, in particolare 275-276). 
ben offenbart ${ }^{34}$ und statt zum gedankenlosen Nachahmen vielmehr zum selbstständigen Nachschöpfen anleitet und begeistert.

Alla luce di questa interpretazione della sentenza ciceroniana ${ }^{35}$, Mommsen nega recisamente che il moderno cesarismo trovi un'anticipazione nella storia di Cesare, la quale è anzi - in realtà («wahrlich») - «eine bittrere ${ }^{36}$ Kritik der modernen Autokratie, als eines Menschen Hand sie zu schreiben vermag». Ed è significativo (in ordine a quanto si è appena osservato, in nota, con riferimento alla citazione immediatamente precedente) che sull'analogia di una «legge di natura» Mommsen fondasse la propria radicale critica dell'assolutismo e la connessa esaltazione del costituzionalismo liberale:

Nach dem gleichen Naturgesetz, weßhalb der geringste Organismus unendlich mehr ist als die kunstvollste Maschine, ist auch jede noch so mangelhafte Verfassung, die der freien Selbstbestimmung einer Mehrzahl von Bürgern Spielraum läßt, unendlich mehr als der genialste und humanste Absolutismus ${ }^{37}$; denn jene ist der Entwickelung ${ }^{38}$ fähig, also lebendig, dieser ist was er ist, also todt.

Ma se la stessa «legge di natura» spiega la successiva degenerazione della monarchia cesariana nell'assolutismo imperiale («Wenn in den Anfängen der Autokratie und vor allem in Caesars eigener Seele [...] noch der hoffnungsreiche Traum einer Vereinigung freier Volksentwicklung und absoluter Herrschaft waltet, so hat schon das Regiment der hochbegabten Kaiser des julischen Geschlechts in schrecklicher Weise

34 Si profila la 'filosofia della storia' di stampo positivistico che avrebbe costituito quanto meno il quadro concettuale della successiva, più ampia e caratteristica, fase dell'operosità scientifica di Mommsen (compreso, si è visto, il V volume della Römische Geschichte).

35 Ad essa faceva non sorprendente riferimento anche Charles Alexandre nell' «Avant-propos» del primo tomo della citata traduzione francese, p. VI: «Nous rencontrons un attrait toujours neuf et puissant dans ces grandes leçons du passé qui nous enseignent le présent, comme les vicissitudes des temps présents nous donnent souvent le secret des événements d'autrefois». Ma è interessante l'aggiunta di Alexandre, segnalata dal corsivo (mio), con la quale la sentenza sfociava nella sentenza inversa, vita magistra historiae, cara a Gaetano De Sanctis, che ne fece un caratteristico principio metodico (rinvio al saggio "Vita magistra historiae". La concezione storica di Gaetano De Sanctis nella Storia dei Romani» (in corso di pubblicazione).

36 Poi (III ${ }^{5}, 1869$, p. 462): «schärfere».

37 È il liberale del XIX secolo, il liberale della Costituzione di Francoforte (1848), che si riconosce nell'icastica spiegazione della superiorità di una (anche se difettosa) costituzione liberale, la quale lasci alla maggioranza dei cittadini l'autodeterminazione del loro destino, rispetto al più geniale ed umano degli assolutismi: la costituzione è suscettibile di sviluppo, è dunque un organismo vivente; l'assolutismo non può cambiare, è cosa morta.

38 Poi (III ${ }^{5}, 1869$, p. 462): «Entwicklung». 
gelehrt, inwiefern es möglich ist in dasselbe Gefäß Feuer und Wasser zu fassen» ${ }^{39}$ ), resta intatto il giudizio conclusivo di Mommsen sull'opera di Cesare:

Caesars Werk war nothwendig und heilsam, nicht weil es an sich Segen brachte oder auch nur bringen konnte, sondern weil, bei der antiken auf Sklaventum gebauten von der republikanisch-konstitutionellen Vertretung völlig abgewandten Volksorganisation und gegenüber der legitimen in der Entwicklung eines halben Jahrhunderts zum oligarchischen Absolutismus herangereiften Stadtverfassung, die absolute Militärmonarchie der logisch nothwendige Schlußstein und das geringste Uebel war.

La monarchia cesariana, "democratica» (si è visto), era dunque anche "necessaria», in quanto «logicamente» imposta da un'organizzazione politica fondata sulla schiavitù e sfociata nell'assolutismo oligarchico; onde la 'previsione' dettata dall'analogia di situazioni storiche pur tanto lontane nel tempo e nello spazio:

Wenn einmal in Virginien und $\mathrm{Ohio}^{40}$ die Sklavenhalteraristokratie es so weit gebracht haben wird wie ihre Wahlverwandten in dem sullanischen Rom, so wird dort auch der Caesarianismus vor dem Geist der Geschichte legitimirt sein; wo er unter andern Entwicklungsverhältnissen auftritt, ist er zugleich eine Fratze und eine Usurpation [...].

\section{4}

Interrompo qui la citazione ${ }^{41}$, perché il riferimento - nell'aggiunta del 1857 - all'aristocrazia schiavistica di alcuni Stati della confederazione nordamericana fu occasione di un'ulteriore aggiunta nella quarta edizione ${ }^{42}$ :

Als dies geschrieben wurde, im Jahre 1857, konnte man noch nicht wissen, wie bald durch den gewaltigsten Kampf und den herrlichsten Sieg, den die Geschichte des

39 Così (segnalando almeno un esempio caratteristico) la convergenza di pensiero politico e arte letteraria di Mommsen concorre significativamente alla formulazione del suo giudizio storico.

40 Poi $\left(\mathrm{III}^{4}, 1866\right.$, p. 463) : « in Virginien und den Carolinas ». Vd. la successiva n. 43.

41 Ma non si può non richiamare l'icastica conclusione: «Die Geschichte aber wird sich nicht bescheiden dem rechten Caesar deßhalb die Ehre zu verkürzen, weil ein solcher Wahlspruch den schlechten Caesaren gegenüber die Einfalt irren und der Bosheit zu Lug und Trug Gelegeheit geben kann. Sie ist auch eine Bibel, und wenn sie so wenig wie diese dem Thoren es wehren kann sie mißzuverstehen und dem Teufel sie zu citiren, so wird beides auch ihr ebensowenig zu schaden im Stande sein».

$42 \mathrm{III}^{4}, 1866$, p. 463 nota (inserita dopo le parole «so wird dort auch der Caesarianismus vor dem Geist der Geschichte legitimirt sein»). 
Menschengeschlechts bisher verzeichnet hat ${ }^{43}$, demselben ${ }^{44}$ diese furchtbare Probe erspart und dessen Zukunft der unbedingten, durch keinen localen Caesarianismus ${ }^{45}$ auf die Dauer zu hemmenden sich selbst beherrschenden Freiheit gesichert werden sollte.

Aveva precorso questa nota di Mommsen - si 'scopre' con sorpresa ed interesse - l'autore della prima traduzione italiana della Römische Geschichte ${ }^{46}$. Nella traduzione del III volume, pubblicata nel 1865 (un anno prima della quarta edizione tedesca), Giuseppe Sandrini faceva infatti seguire alla previsione di Mommsen ("Quando nella Virginia e nell'Ohio l'aristocrazia dei tenitori di schiavi avrà spinto le cose come i suoi emuli nella Roma di Silla, sarà anche colà legittimato il cesarismo dinanzi allo spirito della storia») una pertinente, acuta considerazione ${ }^{47}:$ "La recente emancipazione degli schiavi negli Stati Uniti d'America, non preveduta dal Mommsen, che scriveva il suo volume nel $1854^{48}$, mutò naturalmente la posizione degli antichi proprietarii di schiavi, per cui le conseguenze prevedute dall'autore difficilmente potranno aver luogo». Non si può fare a meno di esprimere la più viva ammirazione per i traduttori di un tempo!

Ho proposto un esempio della convergenza e intrinseca connessione della storiografia, del pensiero politico, dell'arte letteraria di Mommsen nei primi tre volumi della Römische Geschichte, attraverso la lettura di alcune pagine singolarmente efficaci e

43 Ovvio il riferimento alla recente conclusione della Guerra civile americana (1861-1865), alla luce della quale si spiega la correzione di Mommsen citata nella precedente n. 40: l'Ohio era rimasto infatti dalla parte dell'Unione, mentre la Carolina del Sud era stata la prima a proclamare la secessione.

44 Cioè, «dem Menschengeschlecht», opportunamente tradotto «aux États-Unis» da Charles Alexandre (nell'edizione citata nella precedente n. 10: VIII, p. 66 n. 1) e «agli USA» nell'edizione italiana più diffusa (citata nella stessa nota: II, p. 1214 n. 5).

45 Come si è detto nella precedente n. 31, il termine "Caesarismus" avrebbe sostituito "Caesarianismus» solo nella quinta edizione (1869), forse anche in séguito al successo dell'opera di NAPOLÉON III, Histoire de Jules César, I-II, Paris, Imprimerie Impériale (edizione in-folio) e Plon (edizione in-quarto), 1865-1866. Su Mommsen e Napoleone III: CHRIST, Caesar, p. 151-152.

46 T. Mommsen, Storia romana, Prima traduzione dal tedesco di G. Sandrini, I 1, Torino, Guigoni, 1857; I 2, Milano, Guigoni, 1862; II, ivi, 1864; III, ivi, 1865. - Nel 1857, quando usciva la prima parte del I volume, questa traduzione era effettivamente la "prima traduzione dal tedesco» (è uno dei suoi vari motivi d'interesse).

47 Inserita come «Nota del Trad[uttore]» a p. 447.

48 In realtà, dopo il 1856 (data di pubblicazione della prima edizione del III volume): la 'previsione' di Mommsen compare nella seconda edizione (1857). 
significative, sia per la trasparente qualità letteraria dell'esposizione ${ }^{49}$ e la forza concettuale delle caratteristiche riflessioni di carattere politico generale, sia (soprattutto) per il rapporto funzionale che i due aspetti hanno con il preminente aspetto storiografico.

E poiché l'aspetto storiografico dell'esempio proposto si identifica con Cesare, nella duplice prospettiva del più grande personaggio storico dell'antica Roma e di un'ideologia politica del XIX secolo che da lui traeva il nome, la valutazione di questo aspetto presuppone un'analisi comparativa fra la posizione di Mommsen (da una parte) e il successivo svolgimento storiografico di oltre un secolo e mezzo (dall'altra). È quel che, sulla storiografia relativa a Cesare, ha fatto Karl Christ nella sua citata, esemplare indagine ${ }^{50}$. Quanto al cesarismo, un fenomeno politico di origine francese divenuto universale nel corso del XX secolo, si capisce che esso abbia finito per attrarre un'attenzione più politica che storiografica, anzi quasi esclusivamente politica ${ }^{51}$; tanto più si è indotti a rilevare, e ad ammirare, la vigorosa coesistenza di ispirazione storiografica e politica che informa, e ha reso celebre, l'intervento polemico di Mommsen contro l'identificazione di Cesare e cesarismo.

Sui problemi relativi a Cesare e al cesarismo non mi trattengo, anche per lasciare a questo contributo la sostanza della sua presentazione a Tolosa. Sono problemi, tuttavia, che non riguardano solo la storia romana o la storia del XIX secolo; essi costituiscono anche parte essenziale di un contributo di storia della storiografia. Perché la storia della storiografia non dovrebbe mai limitarsi allo studio degli autori o delle opere, tanto più che autori ed opere non si lasciano comprendere (se non superficialmente) senza la duplice considerazione storica sia dell'epoca trattata, sia dell'epoca di appartenenza. Ed

49 In prospettiva letteraria, appunto, non sono senza significato i ritocchi formali apportati da Mommsen nel corso delle varie edizioni dell'opera; anche per questo motivo è sembrato opportuno segnalarli.

50 Christ, Caesar, p. 134-154 (sul Cesare di Mommsen) e 155-315 (sullo svolgimento della storiografia cesariana da Eduard Meyer alla fine del XX secolo). E si ricordano alcuni dei contributi raccolti in K. Christ, E. GabBa (Hg.), Caesar und Augustus (Römische Geschichte und Zeitgeschichte in der deutschen und italienischen Altertumswissenschaft des 19. und 20. Jahrhunderts, I), Como, New Press, 1989.

51 È significativo, in questo senso, l'articolo di A. PANEBIANCO, "Cesarismo», in Enciclopedia delle scienze sociali, I, Roma, Istituto della Enciclopedia Italiana, 1991, p. 714-720, dove l'origine storica del termine è confinata in un paio di righe: «Nonostante l'ispirazione provenga da due differenti epoche storiche e i termini evochino due figure diverse di leader (Giulio Cesare e Napoleone Bonaparte), nel lessico politico contemporaneo cesarismo e bonapartismo sono termini intercambiabili e come sinonimi verranno considerati anche in questo articolo». - Sul problema dell'analogia storica fra il potere di Cesare e il cesarismo dei due Napoleoni (affrontato polemicamente, e negato, da Mommsen), si è ricordato il fondamentale saggio di Momigliano, «Per la storia dell'idea di Cesarismo"; per una sintesi degli essenziali dati storici, storiografici e bibliografici rinvio al mio saggio "Imitatio Caesaris. Cesare e Alessandro, Napoleone e Cesare», in A. BARZANò, C. BEARZOT, F. Landucci, L. Prandi, G. ZeCChini (cur.), Modelli eroici dall'antichità alla cultura europea, Roma, «L'Erma» di Bretschneider, 2003, p. 403-414, in particolare 408-412. 
è così che la storia della storiografia può risolversi in «ricerca storica a più dimensioni», come ebbi occasione di definirla nella recensione di una raccolta di saggi storiografici di Arnaldo Momigliano ${ }^{52}$. Non è certo un risultato frequente, nelle indagini di storia della storiografia; il titolo del mio contributo ha voluto almeno segnalare questa loro suggestiva potenzialità.

Che poi, nel corso del contributo, abbia spesso preferito far 'parlare' Mommsen, non è solo perché le sue parole risultano ovviamente più trasparenti ed efficaci di ogni parafrasi e commento; come dissi a Tolosa (piace rinnovare il gradito ricordo di quella giornata), «j'ai saisi volontiers l'occasion pour rappeler que les classiques de l'historiographie ne sont pas là pour être cités; ils sont là pour être lus».

Leandro Polverini

Via Antistene 5

I-00124 Roma

l.polverini@uniroma3.it

52 Essays in ancient and modern historiography, Oxford, Blackwell, 1977. La recensione è in Athenaeum 68 (1980), p. 480-483; vd. in particolare p. 481: «[...] la ricerca 'storiografica' del M. è, in realtà, ricerca 'storica', e non già (o non solo) nel senso ovvio che la storiografia può costituire oggetto di ricerca storica al pari di qualsiasi altra manifestazione intellettuale o materiale della vita umana; e nemmeno nel senso 'storicistico' che lo studio di un fenomeno culturale, quale è la storiografia, non può in ogni caso prescindere dai presupposti di ordine politico, sociale, economico e dalla loro valutazione storica; ma perché un'indagine come quella del M., dove ad ogni passo si intersecano i piani temporali rispettivamente individuati dall'opera storiografica presa in esame, dal periodo storico in essa trattato, dalle circostanze in cui si svolge - ricavandone sollecitazioni non solo intellettuali - la riflessione dello studioso sull'una e sull'altro, non è che una forma più complessa e articolata (se si vuole, più sofisticata) di ricerca storica: di ricerca storica, per così dire, a più dimensioni». 